\title{
Personalized hypertension management based on serial assessment and telemedicine (PHMA): a cluster randomize controlled trial protocol in Anhui, China
}

\author{
Xingrong Shen ${ }^{1 \dagger}$, Siyi Xiao ${ }^{1 \dagger}$, Rong Liu' ${ }^{1}$, Guixian Tong ${ }^{2}$, Tongzhu Liu² and Debin Wang ${ }^{1 *}$ (i)
}

\begin{abstract}
Background: Despite tremendous investment worldwide, hypertension treatment and control rates remain low. The complexity and long-term dynamics of influencing factors make personalized management inevitable and challenging. This protocol describes Personalized Hypertension Management in Anhui, China (PHMA), a project that uses a package of innovative approaches in tailoring interventions to individual patient's dynamic complications and contexts.
\end{abstract}

Methods/design: PHMA strives to reduce hypertension harms by eight "objective behaviors" (e.g., self-monitoring and reporting, healthy diet, physical exercise/activities). These objective behaviors are promoted through five intervention measures: support for self- monitoring, supervised machine communications, daily education or reminder messages, weekly blood pressure notification, and quarterly signed feedback. PHMA uses ten categories and over 300 variables in selecting and refining intervention procedures and content for individual patients. Efficacy of the intervention package is evaluated using a cluster randomized controlled trial design involving a total of 60 site communities and 3352 hypertension patients. Primary measure for the evaluation is systolic and diastolic blood pressure; while secondary evaluation measures include quality of life (EQ5D-5L), occurrence of hypertension-related complications (such as cerebral hemorrhage, coronary heart disease, myocardial or cerebral infarction), healthcare utilization and scores of objective behaviors.

Discussion: PHMA uses novel, low cost and sustainable approaches to tailor interventions to the dynamic conditions and contexts of individual patients. Unlike contemporary approaches to hypertension management which are mainly population based, each participant patient in PHMA applies a unique intervention package and all messages, feedbacks and other materials sent out to individual patients are different from each other. PHMA is the first project that adopts comprehensive tailoring and if proved effective, it should have important implications for future research, practice and policy-making.

Trial registration ISRCTN10999269. July 17, 2020; https://doi.org/10.1186/ISRCTN10999269.

\footnotetext{
*Correspondence: dbwang@vip.sina.com

${ }^{+}$Xingrong Shen and Siyi Xiao have contributed equally to this work

1 School of Public Health, Anhui Medical University, 81 Meishan Road,

Hefei, China

Full list of author information is available at the end of the article
}

(C) The Author(s) 2021. Open Access This article is licensed under a Creative Commons Attribution 4.0 International License, which permits use, sharing, adaptation, distribution and reproduction in any medium or format, as long as you give appropriate credit to the original author(s) and the source, provide a link to the Creative Commons licence, and indicate if changes were made. The images or other third party material in this article are included in the article's Creative Commons licence, unless indicated otherwise in a credit line to the material. If material is not included in the article's Creative Commons licence and your intended use is not permitted by statutory regulation or exceeds the permitted use, you will need to obtain permission directly from the copyright holder. To view a copy of this licence, visit http://creativecommons.org/licenses/by/4.0/. The Creative Commons Public Domain Dedication waiver (http://creativeco mmons.org/publicdomain/zero/1.0/) applies to the data made available in this article, unless otherwise stated in a credit line to the data. 
Keywords: Cluster randomized controlled trial, Hypertension, Behavioral intervention, Personalized management, China

\section{Background}

The number of patients with hypertension has reached 1.13 billion worldwide [1]. It has increased by $30.0 \%$ since 1990 and is projected to increase to 1.56 billion by 2025 [2]. Hypertension causes heavy disease and economic burden. It is the most important risk factor for stroke, heart disease, renal dysfunction and other major diseases [3] and causes an annual deaths of about 9.4 million (17.8\% of the total deaths) globally [4]. Hypertension epidemic and burden in China are equally serious. Total hypertension patients and annual deaths attributable to the disease in the country amounted to 300 and 2.5 million respectively $[5,6]$. The nation's annual direct medical expenses due to cardiovascular diseases was estimated as 130 billion yuan (or 18.4 billion USD), of which 36.6 billion yuan (or 5.2 billion USD) was for hypertension treatment [7].

Human responses to hypertension can be divided into two main areas, i.e., clinical treatment and behavioral intervention. Clinical responses comprise antihypertension medication and detection and treatment of hypertension complications. Behavioral interventions include medication adherence and lifestyle modifications, e.g., healthy diet, physical exercise, alcohol and tobacco containment, stress and insomnia coping, and family engagement [8-12]. However, the effectiveness of existing efforts falls far from expected. Most published interventions demonstrated only marginal to moderate efficacy $[13,14]$. According to systematic reviews published by The Lancet in 2019, the treatment and control rate in high-income countries was $60.6 \%$ and $36.8 \%$ respectively [3]; and $29.9 \%$ and $10.3 \%$ in low- and middle-income countries [15]. The treatment and control rate in China was $38.3 \%$ and $14.5 \%$ [16].

Although a variety of reasons are attributable to the low treatment and control rates, lack of adequate sensitivity to the real needs of individual patients may play a key role. Contemporary approaches are mainly population rather than individual oriented [17-19]. Population level initiatives are highly feasible since they use only a single protocol for all individuals but suffer from inherent inability in tailoring interventions to the heterogeneous and changing conditions and contexts of individual residents [20]. Both antihypertension treatment and behavior modifications belong to life-time endeavor needing strong persistence under complex and dynamic contexts. This complexity and long-term dynamics make personalized hypertension management (PHM) inevitable since the chance to find identical hypertension determinant systems from different patients decreases exponentially as the number of its influencing factors multiplied by the number of potential variations in each of the factors at different time points increases.

However, PHM requires: a sustained information mechanism capable of constantly collecting and preserving data about relevant status and trajectory of individual patients; a sensitive planning mechanism capable of identifying personalized needs from the serial and multisource data and designing tailored management accordingly; and an effective delivery mechanism capable of implementing the planned interventions on a patientby-patient base. These are almost impractical with traditional or mainly manual hypertension management. Due to rapidly advancing computing and communication technologies, more and more hypertension patients are using electronic tonometer, electronic weight scale, smartphone and other mobile devices in monitoring their blood pressure (BP) and related conditions [21-23]. This protocol describes a novel PHM package in Anhui, China (hereafter referred to as PHMA) which takes advantages of these new development and incorporates practical technologies from mobile and telemedicine, cloud computing, and programmed algorithms and models in a synergetic way in tackling the PHM requirements.

\section{Aim/objectives}

The primary goal of this study is to test the efficacy of PHMA. The study hypothesis is that hypertension patients in the intervention arm will, compared to those in the control condition, demonstrate: (a) lower systolic and diastolic BP: (b) higher scores on objective behaviors including self-monitoring, treatment adherence, healthy diet, physical activities, tobacco/alcohol containment, anxiety/insomnia coping, family engagement; and (c) reduced use of medical care due to hypertension and its complications. A second objective of this study is to identify key facilitators, barriers and corresponding strategies in disseminating and implementing PHMA.

\section{Methods/design \\ Intervention ingredients and tailoring Overall framework}

As shown in Fig. 1, PHMA aims at preventing and reducing hypertension harms by eight objective behaviors including: (a) attending and responding to project 


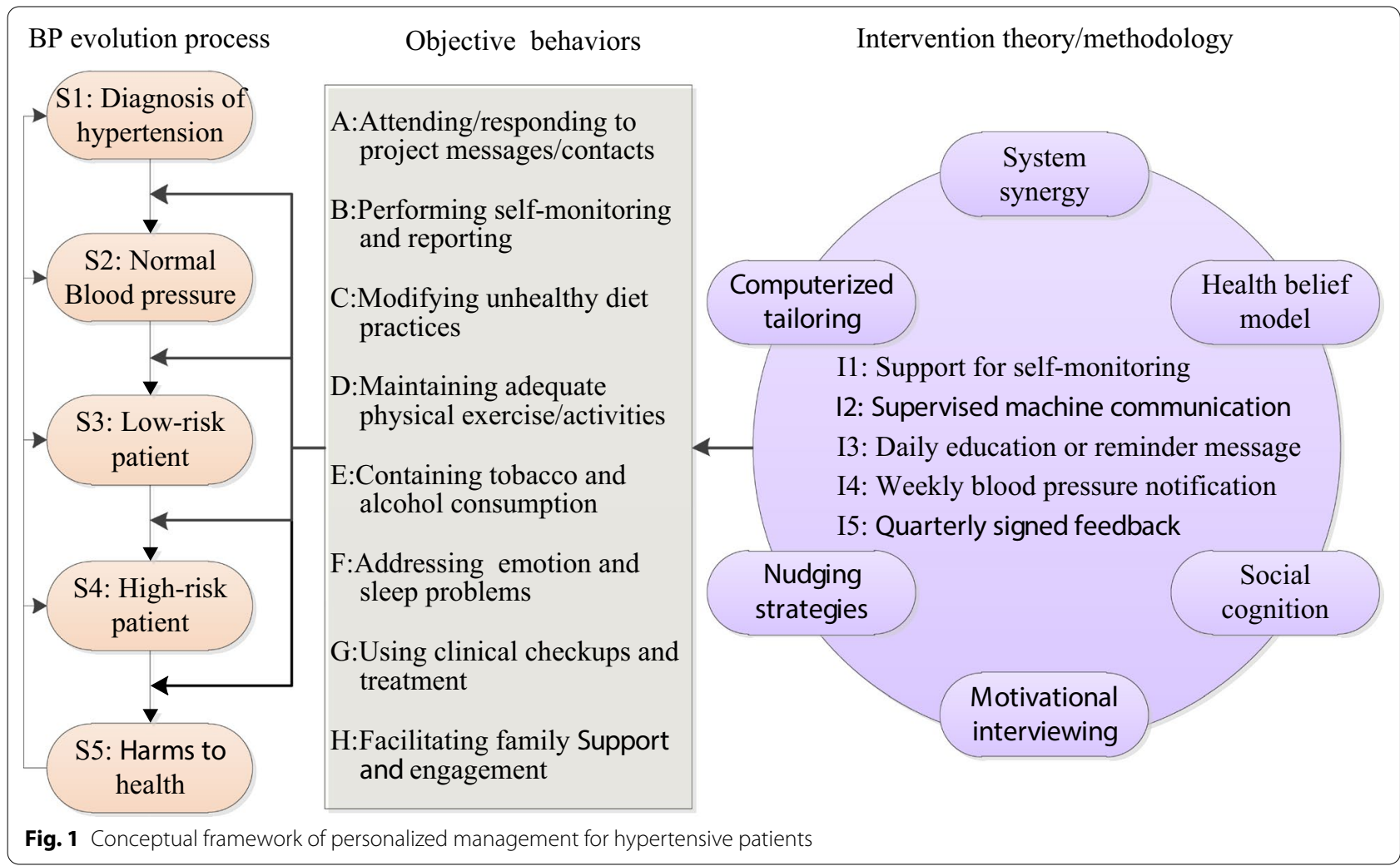

messages/contacts; (b) performing self-monitoring and reporting; (c) modifying unhealthy diet habits or practices; (d) maintaining adequate physical exercise/activities; (e) containing tobacco and alcohol consumption; (f) addressing emotion and sleep problems; (g) using clinical checkups and treatment; and (h) facilitating family engagement. These objective behaviors are promoted through two intervention stages and five intervention measures. More specifically, the intervention for each participating patient starts with an orientation stage followed by a problem-solving stage. The orientation stage aims at equipping the patient with essential knowledge and attitudes about each of the objective behaviors applicable and helping him/her start to practice these behaviors. While the problem-solving stage facilitates the patient to identify and solve problems or barriers encountered in practicing the objective behaviors initiated in the orientation phase. The five intervention measures are: support for self-monitoring (I1), supervised machine communication (I2), daily education or reminder message (I3), weekly blood pressure notification (I4), and quarterly signed feedback (I5). Design of detailed content or procedures of these measures is guided by popular theories or strategies including system synergy [24], health belief model [25], social cognition theory [26], motivational interviewing
[27], nudging strategies [28] and computerized tailoring. The following subsections briefly introduce each of the intervention measures.

\section{Support for self-monitoring (I1)}

PHMA provides necessary technical support for participant patients to facilitate their practice of the objective behaviors especially self-monitoring. First, it maintains a cloud database and web-based support system capable of receiving, storing and processing patients' self-monitored data and allowing them to view their personal records and feedbacks. Second, the project provides each participant patient with an electronic tonometer which: allows the patient to measure point SBP, DBP and pulse at home; sends the results to a cloud data center maintained by the project; and receives and plays voice messages disseminated from the cloud center. Third, the project encourages the participating patients to log onto their personal online accounts to: view their hypertension management profile (Fig. 2) and all the messages, notifications, feedbacks and other IEC (information, education and communication) materials he/she has received in the 


\section{Customer hypertension management profile by: March 3, 2020}

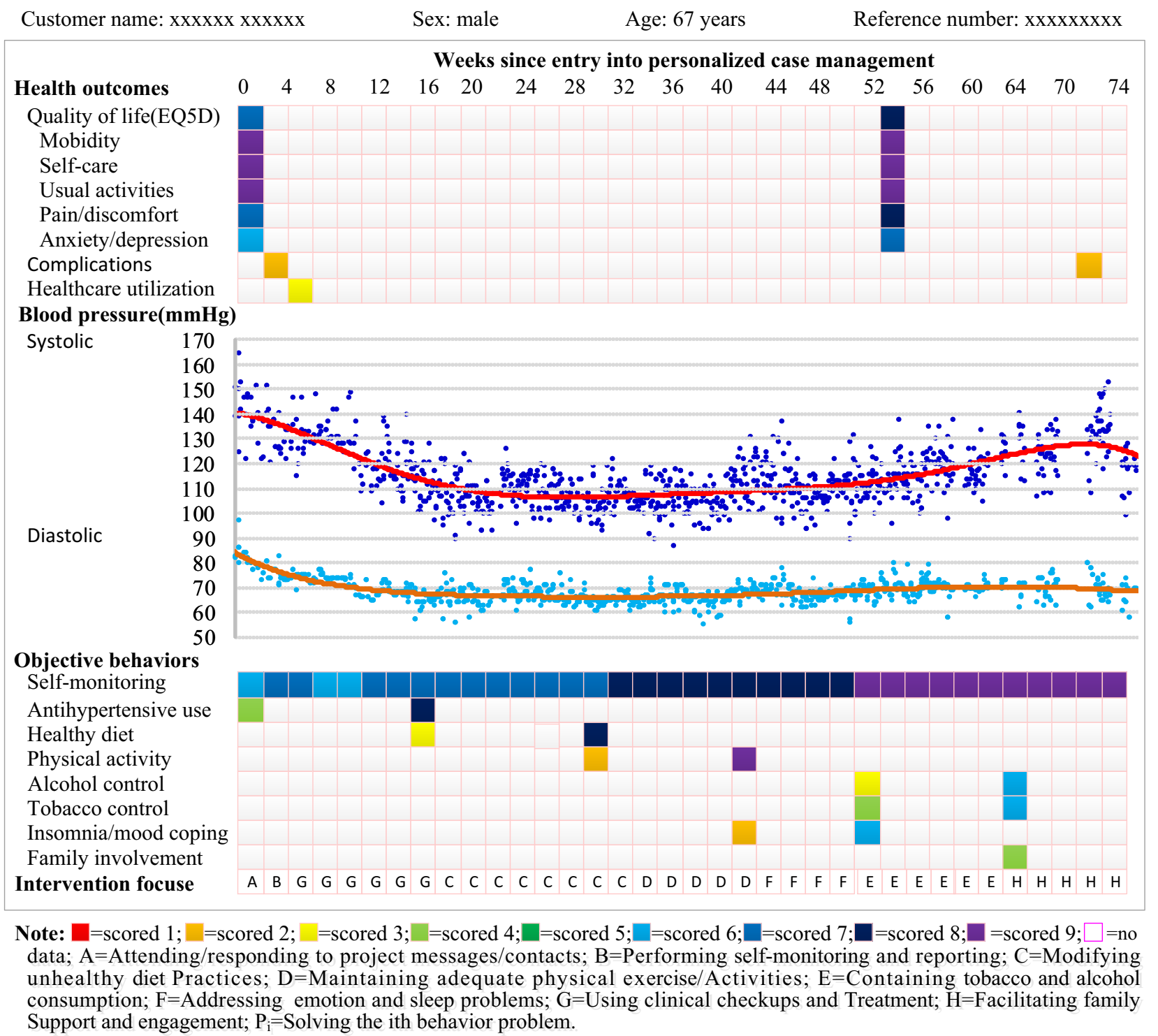

Fig. 2 Example personalized hypertensive management profile

past; and administer relevant self-monitoring questionnaires (Additional file 2: Appendix 1) as suggested.

\section{Supervised machine communication (12)}

PHMA delivers supervised machine communication (SMC) via telephone at the beginning when the patient enrolls into the project and then at the end of every three months. The communication is conducted mainly by an artificial intelligence (AI) voice processing system according to preset transcripts but the process is supervised by a human professional to handle exceptional machine-patient interactions. SMC comprises baseline and follow-up sessions lasting for about half an hour each time. The baseline SMC introduces PHMA and invites participation in the project, and, if successful, performs a brief yet comprehensive assessment of the patient's: (a) diagnosis and treatment history; (b) symptoms of hypertension complications; and (c) BPrelated lifestyle practices. Each of the follow-up SMC session focuses primarily on the patient's hypertension management in the past 3-monthes and discusses: status and trends of his/her BP; efforts and progresses made in managing his/her hypertension; difficulties 
encountered; and plans or suggestions for overcoming the difficulties.

\section{Daily education or reminder message (13)}

PHMA sends short (less than 120 Chinese characters), daily (once a day), and bi-modality (text and voice) messages via: (a) voice electronic tonometer; and (b) WeChat (the most popular social media in China), if the patient is a frequent WeChat user; or (c) mobile phone, if the patient uses mobile phone but are not used to WeChat. Content of the messages differs from patient to patient and from time to time depending on the actual sequence of objective behaviors to be promoted and behavior problems to be addressed. Taking the example shown in Fig. 2, the "intervention focus" during weeks 16-32 was " $\mathrm{C}$ " and so all messages send to that specific patient during these weeks centered on "modifying unhealthy diet". All the messages are purposefully kept short to minimize burden of reading/ listening and a "continued one message every day" strategy is adopted to maintain a consistent bond with the patient and reinforce the objective behavior continuously.

\section{Weekly blood pressure notification (14)}

PHMA sends a weekly notification about trends in SBP, DBP, pulse BP and BP control rate in turn for every participating patient. In other words, if a patient receives a short notification about his/her SBP at the end of this week, he/she will receive a notification about DBP, pulse $\mathrm{BP}$ and $\mathrm{BP}$ control rate at the end of the next one, two and three week(s) respectively. Then these are repeated for every four weeks. Figure 3 uses SBP as an example and illustrates how the content of the notification is generated using the patient's self-measured blood pressure data.

\section{Quarterly signed feedback (I5)}

PHMA disseminates quarterly feedbacks to each of its participant patients via: (a) paper letter sent via postal

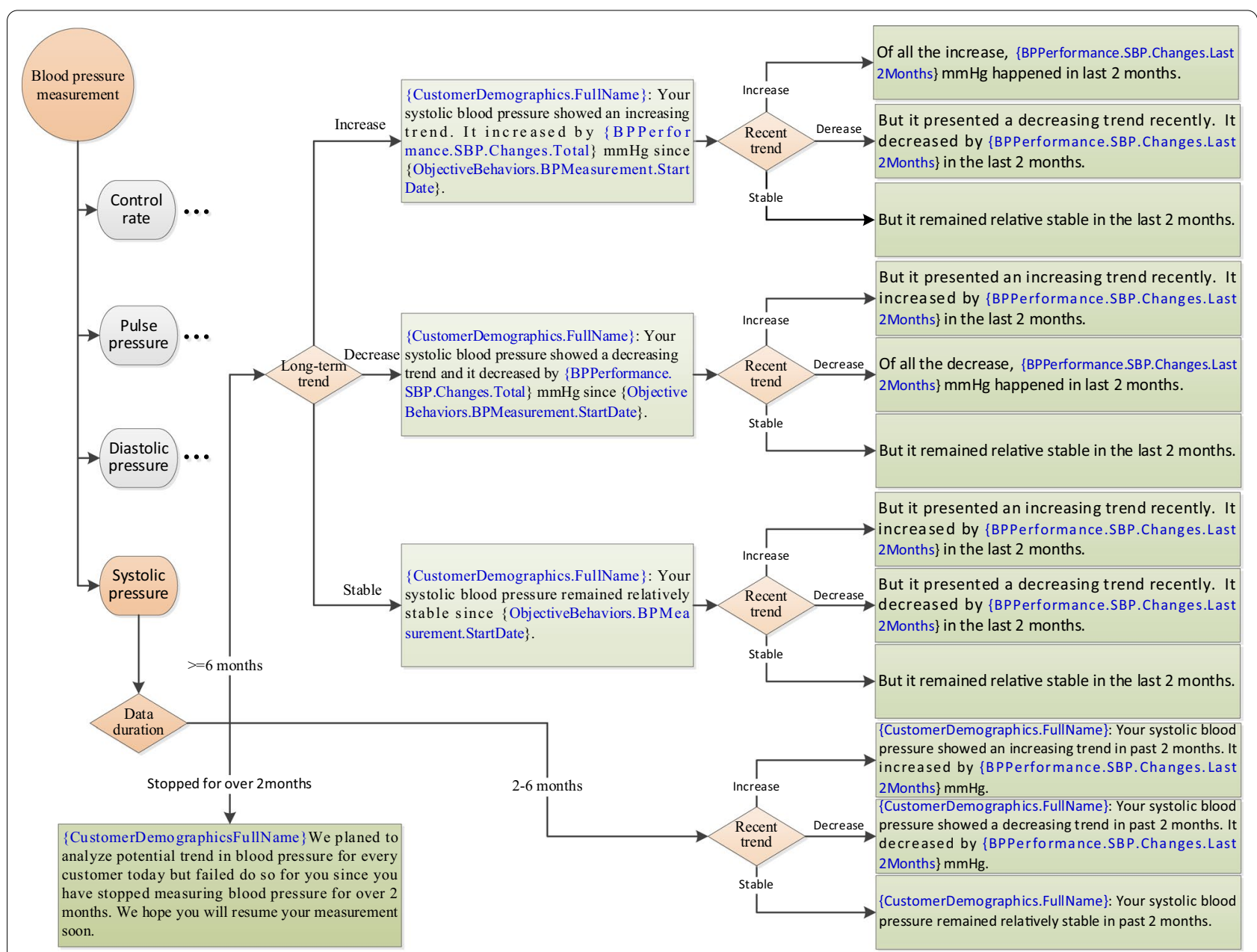

Fig. 3 Example flowchart of how blood pressure notification is generated using patient self-measurements 
service; (b) personal webpage; and (c) WeChat. In the orientation phase, each feedback addresses a specific objective behavior; while in the implementing phase, each feedback addresses a specific barrier to the objective behaviors. All feedbacks comprises five parts: (a) identity and time, to indicate that the feedback is current and addressed to the specific patient named; (b) BP performance, to tell the patient about his/her BP status and trend and indicate that the feedback centers on his/her BP; (c) efforts made recently, to appreciate the patient's efforts and progresses in the previous 3 -months and identify main areas for improvement; (d) actions to take next, to propose feasible actions for the patient to take in the next period; and (e) signatures, to tell the patient that the feedback is produced and checked by authorized professionals and thus useful and reliable. Figure 4 provides an example feedback.

\section{Methods for tailoring interventions}

PHMA uses comprehensive approaches in tailoring interventions to the need and context of individual patients. First, PHMA requires that the intervention for any specific patient in any specific time period focuses on only one most important and feasible objective behavior or behavior problem determined by a priority score. This score is automatically generated by a computerized algorithm based on data accumulated from previous SMC with the patient and his/her self-monitored data. Second, each item in the IEC materials pool of PHMA is designed as a template inserted with variables to be replaced with relevant values, text or diagrams according to the actual conditions/contexts of the patient under concern. The following is a "template" message: "CCustomerDemographics.FullName): Capping monthly salt intake is an effective way in controlling blood pressure. You have $\{\mathrm{Cus}$ tomerDemographics.FamilyMember. Number\} members living together. So, your family should consume less than \{ObjectiveBehavior.HealthyDiet.MonthlyFamilySalt\} grams of salt a month in total." As indicated by "\{\}", this message contains three variables. When sent to a patient named "Zhang San" who has 3 family members (two adults and a child of 8 years), the message is changed into: "Zhang San: Capping monthly salt intake is an effective way in controlling blood pressure. You have 3 members living together. So, your family should consume less than $180 \mathrm{~g}$ of salt a month in total." Fig. 5 shows the template of the quarterly signed feedbacks mentioned earlier and Fig. 4 is an example feedback generated from this template.

\section{Variables for characterizing patients}

PHMA maintains a growing pool of variables for characterizing individual patients. All these variables are classified, coded and computerized in a way that they can be easily: (a) added to relevant IEC templates in intervention development; and (b) replaced, in intervention implementation, with relevant values, text or images according to available data about the specific patient under concern. By the time this paper is written, a total of ten categories and over 300 of variables had been computerized. Table 1 presents all the categories identified so far and example variables under each category.

\section{Study design and settings}

The study adopts a cluster randomized controlled trial (RCT) design involving a total of 60 site communities with 12 in the control arm and 48 in the intervention arm. The control arm maintains existing hypertension case management; while the intervention arms, personalized hypertension management as described above. Project evaluation applies to both arms using the same data collection methods and by same field data collectors. The uneven distribution of site communities between the control and intervention arms was designed to enable detection of potential differences between the control arm and at least four main subgroups with different intervention ingredients within the intervention arm. The analysis and reporting of the trial will strictly follow the CONSORT guidelines [29, 30]. A Trial Steering Committee has been established which holds annual meetings and supervises the trial implementation. For time schedule of the trial please refer to Additional file 1.

\section{Selection and randomization of communities}

The RCT is implemented in Anhui, an inland province located in eastern China with a population of about 70 million living in 105 cities or counties. Selection of participating sites and patients uses a clustered randomization which proceeds in the following steps: step1 divides Anhui province into north, middle and south regions; step 2 randomly selects 4 cities/counties from each of the three regions; step 3 randomly selects five nonadjacent communities from each of the cities/counties selected; step 4 randomly selects 56 patients diagnosed with hypertension from the communities selected. The five communities selected from each city/county in steps 3 are randomly assigned to the control arm $(n=1)$ and intervention arm $(\mathrm{n}=4)$. Hypertensive patients aged 18 years or above and capable and willing to participate in the study from the selected communities meet the inclusion criteria. All the randomization is performed by a statistical professional from outside the project team. The study will be blinded to both data collectors and analyst. 
- Template quarterly feedback

\section{Customer Hypertension Management Feedback Report}

Name: (Concealed)

Date: $2020 / 2 / 28$
Sex: Male

Serial number: 003
Age: 67 years

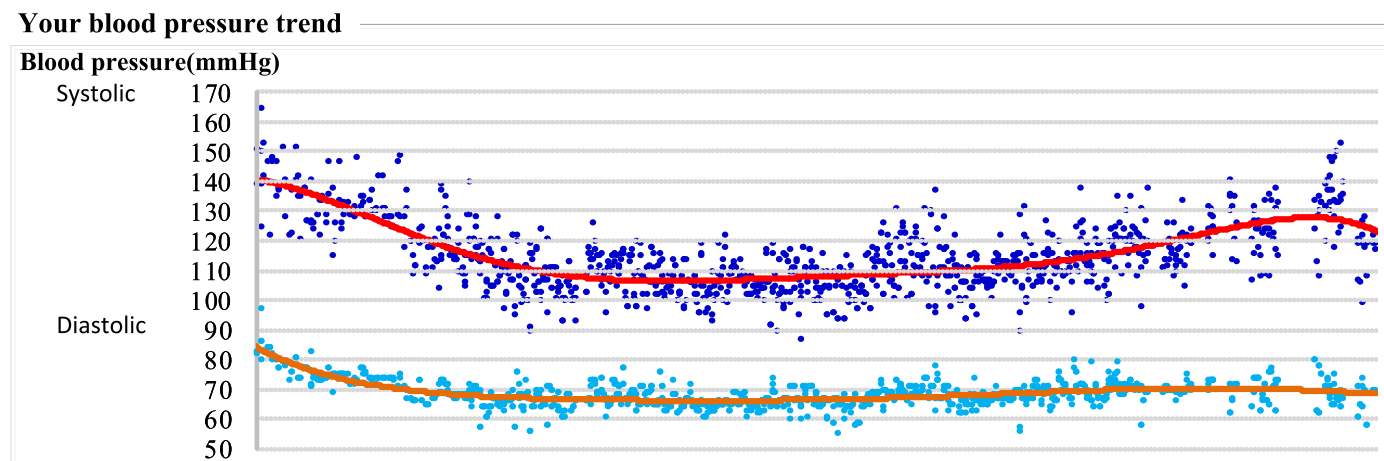

- From November 1, 2018 to date, your self-measured systolic blood pressure decreased by about $15 \mathrm{mmHg}$. But it showed a slight increasing trend in the past two months and increased by about $10 \mathrm{mmHg}$.

- Your self-measured diastolic blood pressure also decreased by some $10 \mathrm{mmHg}$ during the whole time period. But it remained relatively stable in the past two months, being around $70 \mathrm{mmHg}$.

- Over $96 \%$ of your blood pressure measurements were below the "control" threshold, i.e., $140 \mathrm{mmHg}$ for systolic blood pressure and $90 \mathrm{mmHg}$ for diastolic blood pressure. Your blood pressure is being well controlled.

\section{Your hypertension management status}

- We appreciate your efforts in modifying your life style and reducing your complication risks and harms.

- Of the eight objective behaviors we have proposed, you are doing well in use of antihypertensive medications. You take the medicine every day at almost exactly the same time. You have never changed your medication regimen and dose without counseling your doctor.

- You are also keen at self-monitoring. You have been measuring you blood pressure regularly. You answered all our telephone calls and participated sincerely in the communications. You logged into your personal online account from time to time and completed most of the suggested self-assessment.

- However, you have rooms to improve. For example, you are not good at healthy diet. Your salt intake is estimated as over 12 grams a day. You eat pickled meat or vegetables twice a day on average. You seldom consume fruits and coarse grains.

- You also need to improve physical activities. Your are engaged in a sedentary work and you spend most of your leisure time on watching TV and playing Majiong or chards. You seldom do physical exercises.

\section{Your next step suggestions}

Given your blood pressure performance and your current hypertension management, we suggest you focus your attention on healthy diet for the next few months. In particular, please try your best to ensure the following:

- Reduce your salt intake to about 6 grams a day through, presumably, a step by step approach;

- Halve the frequency and amount of your consumption of pickled food;

- Increase your consumption of fresh vegetables to around 500 grams and in at least two meals a day;

- Enrich your consumption of vegetables to at least two kinds a day and four kinds a week;.

- Add coarse grains in all your daily meals;

- Enrich your consumption of coarse grains to at least three kinds a week.

Designed by: (Concealed)

Affiliation: Member of Expert Panel, Personalized Hypertension Management, Anhui

Signature: (Concealed)
Checked by: (Concealed)

Affiliation: Department of Cardiovascular Diseases, the First Affiliated Hospital of Anhui Medical University Signature: (Concealed)

Fig. 4 Example signed quarterly feedback (simplified to fit into one page)

\section{Withdrawal}

Any participant can withdraw from the study at any time. The participants withdrawn from the study will not be replaced but the withdrawal reasons will be recorded. 
— Template quarterly feedback

\section{Customer Hypertension Management Feedback Report}

Name: \{CustomerDemographics.FullName\} Sex:\{CustomerDemographics.Sex $\} \quad$ Age: CustomerDemographics.Age $\}$ Date: $\{$ System.Date $\}$ Serial number: \{ManagementHistory.QuarterlyFeedback.Number\}

Your blood pressure trend

\{BPPerformance.SBP\&DBP.Scatterplot\}

- $\{$ BPPerformance.SBP.Summary\}.

- $\{$ BPPerformance.SBP.Summary\}.

- $\{$ BPPerformance.ControlRate.Summary\}.

\section{Your hypertension management status}

- We appreciate your efforts in modifying your life style and reducing your complication risks and harms.

- Of the eight objective behaviors we have proposed, you are doing well in \{ObjectiveBehavior.Assessment.LastTime. ScoredHighest.Title\}. \{ObjectiveBehavior.Assessment.LastTime.ScoredHighest.HealthyPractice\}.

- You are also keen at \{ObjectiveBehavior.Assessment.LastTime.ScoredSecond.Title\}. \{ObjectiveBehavior.Assessment. LastTime.ScoredSecond. HealthyPractices\}.

- However, you have rooms to improve. For example, you are not good at \{ObjectiveBehavior.Assessment.LastTime. ScoredLeast.Title\}. \{ObjectiveBehavior.Assessment.LastTime. ScoredLeast.HarmfulPractices\}.

- You also need to improve \{ObjectiveBehavior.Assessment.LastTime.ScoredSecondLeast.Title\}. \{ObjectiveBehavior. Assessment.LastTime.ScoredSecondLeast.HarmfulPractices\}.

- Your next step suggestions

Given your blood pressure performance and your current hypertension management, we suggest you focus your attention on \{ObjectiveBehavior.ScoredLeast.Title\} for the next few months. In particular, please try your best to ensure the following:

- $\{$ ObjectiveBehavior.Assessment.LastTime.ScoredLeast.PriorityAction1\}.

- $\{$ ObjectiveBehavior.Assessment.LastTime.ScoredLeast.PriorityAction2\}.

- \{ObjectiveBehavior.Assessment.LastTime.ScoredLeast.PriorityAction3\}..

- $\{$ ObjectiveBehavior.Assessment.LastTime.ScoredLeast.PriorityAction4\}..

- $\{$ ObjectiveBehavior.Assessment.LastTime.ScoredLeast.PriorityAction5\}.

- $\{$ ObjectiveBehavior.Assessment.LastTime.ScoredLeast.PriorityAction6\}..

Designed by: \{Resources.ExpertPanel.MemberInCahrge.FullName\} Checked by: \{Resources.DoctorlnCharge.FullName\} Affiliation: Member of Expert Panel, Personalized Hypertension Management, Anhui

Signature: \{Resources.ExpertPanel.MemberInCahrge.Signature\} Affiliation:\{Resources.DoctorInCharge.Affiliation\}

Signature:\{Resources.DoctorInCharge.Signature\}

Fig. 5 Template signed quarterly feedback

\section{Study sample size}

The above sample size of participating patients is calculated on base of the primary intervention assessment measures, i.e., changes in SBP. Based on our previous study results and our aim of comparing the effects of at least four combinations of main intervention ingredients, we suppose: (a) SBP reduction was about $5.0 \mathrm{mmHg}$ as compared between the intervention and control arms; (b) standard deviation of the SBP reduction was $7.8 \mathrm{mmHg}$; (c) ICC value was 0.05 ; and (d) patients in the intention arm need to be assigned to four subgroups to receive four different combinations of interventions. So, to detect a possible absolute difference of $5.0 \mathrm{mmHg}$ with $90 \%$ power and alpha 0.05 , we need 228 patients in each arm. By allowing for a $20 \%$ attrition rate and a design effect of 2.45 , the total sample size is estimated as $3352(=2.45 * 228 * 5 * 1.2)$ and this translates into 56 patients per community.

\section{Measures}

The primary measures for assessing the efficacy of PHMA are SBP/DBP. The secondary measures include quality of life (EQ5D-5L), hypertension-related complications, healthcare utilization and objective behaviors.

\section{Data collection and management}

All the above measures together with data about potential confounding variables (e.g., age, sex, education, years since hypertension diagnosis) are collected at the patients' households by trained data collectors at baseline and 12 and 24 months after baseline. SBP/DBP is measured using a mercury sphygmomanometer in accordance with standard operation specifications [31]; while the remaining data are solicited using structured questionnaires (Additional file 2) administered face-to-face by the 
Table 1 Categories and examples of variables used to tailor interventions

Demographics of patients (CustomerDemographics)

- Patient's full name (CustomerDemographics. FullName);

- Patient's Sex (CustomerDemographics.Sex);

- Patient's Family name (CustomerDemographics. FamilyName);

......

Status and trend of blood pressure (BPPerformance)

- Overall mean of SBP (BPPerformance.SBP.Mean.Overall);

- Mean of SBP for the last 10 times (BPPerformance.SBP.Mean.Last10Times);

- Overall trend of DBP in past year (BPPerformance.DBP.Trend.LastYear);

Patient outcome (PatientOutcome)

- Score of quality of life (EQ5D) as assessed last time (PatientOutcome.QoL.LastAssessment.Score);

- Date when quality of life was last assessed (PatientOutcome.QoL LastAssessment.Date);

- Complications occurred in the past year (PatientOutcome.Complications.LastYear. All);

$$
\text { ...... }
$$

Clinical history in relation to hypertension (ClinicalHistory)

- Year in which hypertension was diagnosed (ClinicalHistory.Hypertension.Diagnosis.Year);

- Whether medication treatment is in use (ClinicalHistory.Hypertension.Treatment.Current.Status);

- Type of current medications (ClinicalHistory.Hypertension.Treatment.Current.Type);

.......

Objective behaviors (ObjectiveBehaviors)

- Title of the objective behavior scored the highest in previous assessment (ObjectiveBehaviors.LatestAssessment. ScoredHighest.Title);

- Score of "healthy diet" in the latest assessment (ObjectiveBehaviors.LatestAssessmen.HealthyDiet.Score);

- Title of objective behavior scored the least in latest assessment (ObjectiveBehaviors.LatestAssessment. ScoredLeast.Title);

$$
\text { -..... }
$$

Problems with or barriers to objective behaviors (BehaviourProblems)

- List of problems or barriers identified from the latest assessment (BehaviourProblems.LatestAssessment.FullList);

- Title of problem scored the highest in the latest assessment (BehaviourProblems.LatestAssessment. ScoredHighest.Title);

- Description of problem scored the highest in the latest assessment (BehaviourProblems.LatestAssessment. ScoredHighest.Description);

......

Hypertension-related physical indicators (Physicallndicator)

- Body weight as measured last time (Physicallndicator.BodyWeight.LastMeasurement.Value);

- Date when patient body was last weighted (Physicallndicator.BodyWeight.LastMeasurement.Date);

- Waist circumference as measured last time (Physicallndicator.WeistCircumference.LastMeasurement.Value);

......

Daily activities (DailyActivity)

- Common working activities as reported last time (DailyActivity.LastReport.Work.Description);

- Common working hours as reported last time (DailyActivity.LastReport.Work.Hours);

- Common leisure activities as reported last time (DailyActivity.LastReport.Leisure.Description):"

......

Social relations (SocialRelations)

- Number of family members (SocialRelations.FamilyMember.Number);

- Member of family members (SocialRelations.FamilyMember.RelationList);

- Family member in closest contact with the patient (SocialRelations.FamilyMember.InClosestContact.Relations);

$$
\text { ...... }
$$

Resources relating to hypertension management (Resources)

- Family income per year (Resources.Faimly.Annuallncome)

- Minutes needed to get to the nearest hypertension clinic (Resources.MinutesToNearestClinic);

- Name of doctor in charge of the patient (Resources.DoctorlnCharge.Current.FullName); 
Table 1 (continued)

"()" defines the start and end of the code of the variable or variable category before it

field data collectors. Data collected at the household will be double entered using EPI DATA V3.1.

Study documentation will be kept in locked files in the offices of the project assessment supervisor and the Principal Investigator. All data will be backed-up on separate media and stored in a secure filing cabinet. Access to security passwords will be given only to the Principal Investigator and the Assessment Supervisor. Personal identifiers will not be stored in the data set and all computers will be protected by antivirus software. These data safety and confidentiality procedures are overseen by a Data Monitoring Committee.

\section{Data analysis}

The data collected will be used to compare the differences between the control groups and the intervention group as a whole and subgroups with different intervention ingredients in the intervention arm at different time points in terms of: (a) SBP/DBP; and (b) the secondary measures including quality of life, occurrence of hypertension-related complications, healthcare utilization, and scores of objective behaviors. Estimation of statistical significance and confidence intervals will assume a type I error established in alpha $=0.05$, using the IBM SPSS V22 statistics package. Despite our anticipated high follow-up rates, we expect some extent of missing data, typically participants missing an interview and are then found at the next follow-up assessment. We will use appropriate statistical techniques to estimate the missing values and then perform the analysis on the completed sample.

Initial data analysis will consist of descriptive summaries intended to examine the patterns of the various measurements and check for normality of the continuous variables. Necessary transformations will be explored and selected, if necessary, to induce approximate normality. Regarding the numerical variables between two groups, t-test of independent samples for mean comparisons will be carried out. We will also employ generalized hierarchical linear models. These models will include multiple linear regression, as well as binary, ordinal, or nominal logistic regression. In these multilevel analyses, patientspecific measurements are included in the models.

\section{Adverse events}

In this study, the most likely anticipated incident is the loss of privacy, so the identification information of patients will not be placed on response forms but use an unique reference number instead.

\section{Dissemination}

Findings from the study will be summarized for the benefit of public health policymakers, health professionals and health service managers in China and elsewhere. A final workshop will be held for the purpose of presenting the evidence generated by this study and working with local and regional stakeholders to translate this evidence into public health action.

\section{Discussion}

PHMA introduces innovative approaches to tailor interventions to the complex and dynamic $\mathrm{BP}$ and related complications and contexts of individual patients. Contemporary approaches to hypertension management are mainly population-based though some studies have tried some extent of differentiated interventions, e.g., send a reminder to patients who have failed to attend an appointment [32]. PHMA uses ten categories of variables in selecting and refining intervention procedures and content for individual patients. As a result, each participant patient in PHMA applies a unique intervention package and all messages, feedbacks and other materials sent out to individual patients are different from each other. To our knowledge, PHMA is the first project that adopts so comprehensive tailoring and if proved effective, it should have important implications for future research, practice and policy-making.

PHMA adopts pragmatic strategies in securing feasibility and containing cost of interventions. It uses multiple modalities (e.g., text and sound) and venues (e.g., telephone, WeChat, Webpages, paper leaflets) in forming and disseminating intervention materials to suit various patients, especially old and illiterate ones. It strives to leverage mobile devices, self-monitoring, distant communications and computerized programs into effective, low cost, convenient and sustainable interventions. Taking the example of SMC, it may lead to a novel and cost-effective mode of interactions between patients and health professionals. Although a human professional is required to supervise the process of every SMC session, his/her workload is reduced to a large extent as compared with traditional patient-doctor communications. In a traditional communication, the doctor (or other human professional) needs to complete a whole range of activities, e.g., reviewing the patient's status and history, forming an outline plan, and interacting with the patient; while in a SMC session, what the doctor needs to do is only to start the conversation by a brief introduction and then listen to the machine-patient interactions and add occasional 
queries or comments. In addition to workload reduction for the doctor, SMC may also exhibit good quality and experience for the patient, since the procedures and content are preset by an expert panel and then refined by computerized algorithms according to the actual conditions of the patient under concern.

PHMA incorporates guidance from multiple theories. As illustrated in Fig. 1, design of detailed intervention content or procedures is guided by health belief model, social cognition theory, motivational interviewing, nudging strategies and system synergy. All these theories/ strategies have been used successfully in a variety of populations and settings [33-35]. Theory-informed intervention design prevents omission of important aspects and thus enhances overall efficacy [34, 36]. Each of the theories used in PHMA has its own strengths: health belief model is useful in addressing behaviors driven by rational thinking; nudging strategies apply to behaviors triggered by automatic cognitive processes [25, 28]; motivational interviewing is keen at generating and maintaining adequate momentum to leverage sustained behavior changes; system synergy informs incorporation of all intervention ingredients in a way that prevents equifinality and maximizes cost-effectiveness [27, 35, 37].

\begin{abstract}
Abbreviations
PHMA: Personalized hypertension management; SMC: Supervised machine communication; BP: Blood pressure; SBP: Systolic blood pressure; DBP: Diastolic blood pressure; IEC: Information, education and communication; RCT: Randomized controlled trial.
\end{abstract}

\section{Supplementary Information}

The online version contains supplementary material available at https://doi. org/10.1186/s12872-021-01943-5.

Additional file 1. Schedule of enrolment, interventions, and assessments of participants.

Additional file 2. Selected patient assessment questionnaires and temporary scoring systems.

Additional file 3. Patient Consent Record \& Contact Details.

\section{Acknowledgements}

Not applicable

\section{Authors' contributions}

XS and SX contributed equally in conceiving this project and drafting this manuscript. RL developed the web aid. GT and TL facilitated project implementation. DW provided expertise for overall design of the study, and revised and finalized the manuscript. All authors have read and approved the manuscript.

\section{Funding}

This study is externally supported by the National Natural Science Foundation of China (Grant No.: 72004002). The funding source has not plaid any role in the study design, analysis or in the decision to submit the manuscript for publication.
Availability of data and materials

Not applicable.

\section{Declarations}

\section{Ethics approval and consent to participate}

The study protocol had been reviewed and approved by Anhui Medical University Biomedical Ethics Committee (ref: 20200936). This approved protocol clearly states: (a) participation and withdrawal from the study is voluntary; (b) at the beginning of recruitment, a consent from (Additional file 3) will be signed (for those who are literate) or ticked (for those who are illiterate) by the participants; and (c) confidentiality of all informant information is strictly enforced.

\section{Consent for publication}

Not applicable.

\section{Competing interests}

The authors declare that they have no competing interests.

\section{Author details}

${ }^{1}$ School of Public Health, Anhui Medical University, 81 Meishan Road, Hefei, China. ${ }^{2}$ The First Affiliated Hospital of USTC, 17 Lujiang Road, Hefei, China.

Received: 29 January 2021 Accepted: 3 March 2021

Published online: 12 March 2021

\section{References}

1. World Hypertension Day 2019: World Health Organization; 2019. https:// www.who.int/news-room/events/world-hypertension-day-2019.

2. Zhou B, Bentham J, Di Cesare M, Bixby H, Danaei G, Cowan MJ, Paciorek CJ, Singh G, Hajifathalian K, Bennett JE, Taddei C. Worldwide trends in blood pressure from 1975 to 2015: a pooled analysis of 1479 populationbased measurement studies with 19.1 million participants. The Lancet. 2017;389(10064):37-55.

3. Zhou B, Danaei G, Stevens GA, Bixby H, Taddei C, Carrillo-Larco RM, Solomon B, Riley LM, Di Cesare M, lurilli ML, Rodriguez-Martinez A. Long-term and recent trends in hypertension awareness, treatment, and control in 12 high-income countries: an analysis of 123 nationally representative surveys. The Lancet. 2019;394(10199):639-51.

4. A global brief on hypertension: silent killer, global public health crisis: World Health Day 2013: World Health Organization; 2013. https://apps. who.int/iris/handle/10665/79059.

5. Committee of Hypertension Management for Primary Care Physicians in China. China national guidelines for the management of hypertension in grassroots settings. Chin Circul J. 2017;32(11):1041-8.

6. Forouzanfar MH, Afshin A, Alexander LT, Anderson HR, Bhutta ZA, Biryukov $S$, et al. Global, regional, and national comparative risk assessment of 79 behavioural, environmental and occupational, and metabolic risks or clusters of risks, 1990-2015: a systematic analysis for the Global Burden of Disease Study 2015. The Lancet. 2016;388(10053):1659-724.

7. Chen WW, Gao RL, Liu LS, Zhu ML, Wang W, Wang YJ, et al. China cardiovascular disease report 2014. Chin Circ J. 2015;30(7):617-22.

8. Caligiuri SPB, Pierce GN. A review of the relative efficacy of dietary, nutritional supplements, lifestyle, and drug therapies in the management of hypertension. Crit Rev Food Sci Nutr. 2017;57(16):3508-27.

9. Li SJ. Compliance and self-efficacy of drug treatment in community hypertension patients. Chin Gen Pract. 2017;20(S2):367-9.

10. Liu T, Liu X, Li SL, Liu GL. Impact of trans-theoretical model based home visiting on medication compliance behavior in community elderly hypertension patients. Chin J Nurs. 2016;51(5):629-34.

11. Sharman JE, Howes FS, Head GA, McGrath BP, Stowasser M, Schlaich M, et al. Home blood pressure monitoring: Australian expert consensus statement. J Hypertens. 2015;33(9):1721-8.

12. Yang $T T$, Tong $Y Q$, Yin XX, Lu ZX, Gong YH. Evaluation of Morisky medication adherence scale and family APGAR applied to patients with tuberculosis. Chin J Soc Med. 2016;33(6):580-2.

13. Danaei G, Finucane MM, Lin JK, Singh GM, Paciorek CJ, Cowan MJ, et al. National, regional, and global trends in systolic blood pressure since 
1980: systematic analysis of health examination surveys and epidemiological studies with 786 country-years and 5.4 million participants. The Lancet. 2011;377(9765):568-77.

14. Melgarejo JD, Maestre GE, Thijs L, Asayama K, Boggia J, Casiglia E, et al. Prevalence, treatment, and control rates of conventional and ambulatory hypertension across 10 populations in 3 continents. Hypertension. 2017;70(1):50-8.

15. Geldsetzer P, Manne-Goehler J, Marcus M-E, Ebert C, Zhumadilov Z, Wesseh CS, et al. The state of hypertension care in 44 low-income and middle-income countries: a cross-sectional study of nationally representative individual-level data from 1.1 million adults. The Lancet. 2019;394(10199):652-62.

16. Yu DM, Li SJ, Ju LH, GuO QY, Xu XL, Fu P, et al. Status of hypertension awareness, treatment and control among adults in China in 2010-2012. J Hyg Res. 2019;48(6):913-8.

17. Jaffe MG, Lee GA, Young JD, Sidney S, Go AS. Improved blood pressure control associated with a large-scale hypertension program. JAMA. 2013;310(7):699-705.

18. Luders S, Schrader J, Schmieder RE, Smolka W, Wegscheider K, Bestehorn K. Improvement of hypertension management by structured physician education and feedback system: cluster randomized trial. Eur J Cardiovasc Prev Rehabil. 2010;17(3):271-9.

19. Mohan S, Jarhyan P, Ghosh S, Venkateshmurthy NS, Gupta R, Rana R, et al. UDAY: a comprehensive diabetes and hypertension prevention and management program in India. BMJ Open. 2018;8(6):e015919.

20. Fortuna RJ, Rocco TA, Freeman J, Devine M, Bisognano J, Williams GC, et al. A community-wide quality improvement initiative to improve hypertension control and reduce disparities. J Clin Hypertens (Greenwich). 2019;21(2):196-203.

21. Carey RM, Muntner P, Bosworth HB, Whelton PK. Prevention and control of hypertension: JACC health promotion series. J Am Coll Cardiol. 2018;72(11):1278-93.

22. Omboni S, Ferrari R. The role of telemedicine in hypertension management: focus on blood pressure telemonitoring. Curr Hypertens Rep. 2015;17(4):21.

23. Silva BM, Rodrigues JJ, de la Torre DI, Lopez-Coronado M, Saleem K. Mobile-health: a review of current state in 2015. J Biomed Inform. 2015;56:265-72.

24. Wu B, Arranz J, Du J, Zhou D, Traulsen A. Evolving synergetic interactions. J R Soc Interface. 2016;13(120):20160282.

25. Park D-Y. Utilizing the Health Belief Model to predicting female middle school students' behavioral intention of weight reduction by weight status. Nutr Res Pract. 2011;5(4):337.

26. Bandura A. Health promotion from the perspective of social cognitive theory. Psychol Health. 1998;13(4):623-49.
27. Salvo MC, Cannon-Breland ML. Motivational interviewing for medication adherence. J Am Pharm Assoc (2003). 2015;55(4):e354-361; quiz e362-353.

28. Fisher B, Thaler RH, Sunstein CR. Nudge: improving decisions about health, wealth, and happiness. Environ Resour Econ. 2010;47(1):149-50.

29. Moher D, Hopewell S, Schulz KF, Montori V, Gotzsche PC, Devereaux PJ, et al. CONSORT 2010 explanation and elaboration: updated guidelines for reporting parallel group randomised trials. Int J Surg. 2012;10(1):28-55.

30. Schulz KF, Altman DG, Moher D, Group C. CONSORT 2010 statement: updated guidelines for reporting parallel group randomized trials. Ann Intern Med. 2010;152(11):726-32.

31. Hemmelgarn BR, McAlister FA, Grover S, Myers MG, McKay DW, Bolli P, et al. The 2006 Canadian hypertension education program recommendations for the management of hypertension: part I—blood pressure measurement, diagnosis and assessment of risk. Can J Cardiol. 2006;22(7):573-81.

32. Ashoorkhani M, Bozorgi A, Majdzadeh R, Hosseini H, Yoonessi A, Ramezankhani A, et al. Comparing the effectiveness of the BPMAP (Blood Pressure Management Application) and usual care in self-management of primary hypertension and adherence to treatment in patients aged 30-60 years: study protocol for a randomized controlled trial. Trials. 2016;17(1):511.

33. Arno A, Thomas S. The efficacy of nudge theory strategies in influencing adult dietary behaviour: a systematic review and meta-analysis. BMC Public Health. 2016;16:676.

34. Barry HE, Bedford LE, McGrattan M, Ryan C, Passmore AP, Robinson AL, et al. Improving medicines management for people with dementia in primary care: a qualitative study of healthcare professionals to develop a theory-informed intervention. BMC Health Serv Res. 2020;20(1):120.

35. Lindson-Hawley N, Thompson TP, Begh R. Motivational interviewing for smoking cessation. Cochrane Database Syst Rev. 2015;3:CD006936.

36. Ntoumanis N, Ng JYY, Prestwich A, Quested E, Hancox JE, ThøgersenNtoumani C, et al. A meta-analysis of self-determination theory-informed intervention studies in the health domain: effects on motivation, health behavior, physical, and psychological health. Health Psychol Rev. 2020:1-31.

37. Duan $X Y$, Sun $Y$, An XL, Feng Q, Pan DH. Application effect of synergy theory-centered rehabilitation nursing on nursing of patients with peripheral facial paralysis. Biomed Res. 2017;28(12):5300-3.

\section{Publisher's Note}

Springer Nature remains neutral with regard to jurisdictional claims in published maps and institutional affiliations.
Ready to submit your research? Choose BMC and benefit from:

- fast, convenient online submission

- thorough peer review by experienced researchers in your field

- rapid publication on acceptance

- support for research data, including large and complex data types

- gold Open Access which fosters wider collaboration and increased citations

- maximum visibility for your research: over $100 \mathrm{M}$ website views per year

At BMC, research is always in progress.

Learn more biomedcentral.com/submissions 